\title{
Colonization of toxigenic Clostridium difficile among ICU patients: a prospective study
}

Xiaoxia Zhang ${ }^{1,2+}$, Xiaohui Wang ${ }^{1,2+}$, Jingyu Yang ${ }^{1,2}$, Xiaohua Liu' ${ }^{1,5}$, Lin Cai ${ }^{4}$ and Zhiyong Zong ${ }^{1,2,3^{*}}$

\begin{abstract}
Background: A prospective study was performed to investigate the prevalence of colonization among ICU patients and to examine whether asymptomatic carriers were the source of subsequent $C$. difficile infection (CDI) and acquisition of toxigenic C. difficile.

Methods: Rectal swabs were collected from adult patients on admission to and at discharge from a 50-bed medical ICU of a major referral hospital in western China, from August to November 2014. Stools were collected from patients who developed ICU-onset diarrhea. Both swabs and stools were screened for $t c d B$ (toxin B gene) by PCR. Samples positive to $t c d B$ were cultured for $C$. difficile and isolates recovered were screened for $t c d B$ and the binary toxin genes by PCR. Strain typing was performed using multilocus sequence typing and isolates belonging to the same sequence type (ST) were further typed using multiple-locus variable number tandem repeat analysis (MLVA).
\end{abstract}

Results: During the 4-month period, rectal swabs were collected from 360 (90.9\%) out of 396 patients who were admitted to the ICU. Among the 360 patients, 314 had stayed in the ICU more than 3 days, of which 213 (73.6\%) had a rectal swab collected within the 3 days prior to discharge from ICU. The prevalence of toxigenic $C$. difficile colonization was $1.7 \%$ (6 cases) and $4.3 \%$ (10 cases) on admission and discharge, respectively. Only four (1.1\%) out of 360 patients had CDI, corresponding to 10.7 cases per 10,000 ICU days. None of the four cases had toxigenic C. difficile either on admission or at discharge. Toxigenic $C$. difficile isolates were recovered from all swabs and stool samples positive for $t c d B$ by PCR and belonged to 7 STs (ST2, 3, 6, 37, 54, 103 and 129). None of the isolates belonging to the same ST had identical MLVA patterns. Binary toxin genes were detected in one ST103 isolate that caused colonization.

Conclusion: The prevalence of colonization with toxigenic C. difficile among patients on admission to ICU was low in our setting. ICU-acquired toxigenic $C$. difficile were not linked to those detected on admission. Active screening for toxigenic $C$. difficile may not be a resource-efficient measure in settings with a low prevalence of colonization.

Keywords: Clostridium difficile, Colonization, Active screening, ICU, MLST, MLVA

\section{Background}

Toxigenic Clostridium difficile has been well recognized as a major pathogen causing healthcare-associated infections in western countries [1]. In healthcare settings, $C$. difficile can be transmitted via contact with patients or their environment directly or indirectly. Contact precautions are

\footnotetext{
* Correspondence: zongzhiy@scu.edu.cn

${ }^{\dagger}$ Equal contributors

${ }^{1}$ Center of Infectious Diseases, West China Hospital, Sichuan University, Guoxuexiang 37, Chengdu 610041, China

${ }^{2}$ Division of Infectious Diseases, State Key Laboratory of Biotherapy, Chengdu, China

Full list of author information is available at the end of the article
}

therefore required when $C$. difficile infection (CDI) is suspected $[2,3]$. Some patients can be colonized with toxigenic C. difficile but without manifestations of CDI. These asymptomatic carriers may serve as an important reservoir of toxigenic C. difficile [4] and appear to have significant higher risks of subsequent CDI [5]. Active screening patients for toxigenic $C$. difficile may therefore have two benefits. One is to identify the source of this pathogen for subsequent control measures, such as contact precautions for carriers, and another is to identify the population at high risk of CDI. However, active screening is resourceintensive and requires corresponding laboratory capacity, 
which may be problematic in developing countries like China. In settings with a high prevalence of colonization with toxigenic $C$. difficile and where nosocomial transmission is frequently linked to asymptomatic carriers, it may be justified to include active screening in the prevention and control of CDI. There are a few studies on the prevalence of colonization for inpatients and residents in longterm care facilities or nursing homes [5, 6]. However, colonization with toxigenic $C$. difficile among ICU patients remains largely uninvestigated. In addition, little is known about the carriage and transmission of toxigenic $C$. difficile in China. We therefore performed a prospective study to investigate the prevalence of colonization with toxigenic $C$. difficile among patients in a large ICU of a major teaching hospital in China and to examine whether the toxigenic $C$. difficile isolates acquired during the ICU stay were linked to asymptomatic carriers who were colonized on admission to the unit.

\section{Methods}

\section{Patient enrollment and sample collection}

This prospective study was conducted among adult patients (age $\geq 16$ ) at a 50-bed medical ICU ward in West China Hospital, Sichuan University, the major referral medical center in western China, during a 4-month period from August to November 2014. This study was conducted in accordance with the amended Declaration of Helsinki and was approved, under a waiver of consent, by the Ethics Committee of West China Hospital. Stool samples and rectal swabs were taken as part of routine care as collecting stool specimens or rectal swabs for screening multidrug resistant organisms such as extended-spectrum $\beta$-lactamase-producing Enterobacteriaceae and vancomycin-resistant enterococci has been a routine practice in the ICU.

Rectal swabs were collected from patients within 2 days of admission to the ICU and also within the 3 days prior to ICU discharge for those patients with a length of stay of 3 days or more. Swabs were transferred to the laboratory in transport media. Stool samples were also collected from patients who developed ICU-onset diarrhea with naturally-passed faeces that were defined as unformed at least 3 times a day.

The presence of toxigenic $C$. difficile in a rectal swab or stool sample was detected by PCR for the toxin Bencoding gene $t c d B$ of $C$. difficile (see below). A carrier was defined as a patient without diarrhea whose rectal swab was positive for toxigenic $C$. difficile. CDI here was defined as diarrhea plus the presence of toxigenic $C$. difficile in stool, in the absence of other reasonable causes of diarrhea. Screening results were not shared with ICU clinicians and infection control nurses and no contact precautions were taken for carriers of toxigenic $C$. difficile.

\section{Screening, culture and PCR confirmation}

Swabs and stool samples were processed immediately after arrival at the laboratory. Total DNA was prepared from swabs and stool using the Stool DNA Kit (OMEGA, Norcross, GA) and was screened for the species-specific gene tpi (encoding triose phosphate isomerase of $C$. difficile) and $t c d B$ of $C$. difficile by PCR as described previously $[7,8]$.

Samples positive for both tpi and $t c d B$ were cultured anaerobically. Briefly, samples were treated with an equal volume of $95 \%$ ethanol before streaking onto cefoxitin cycloserine fructose agar (CCFA; OXOID, Basingstoke, UK) plates complemented with the CDMN supplement (OXOID) and $5 \%$ sheep blood. Plates were incubated in anaerobic jars at $37{ }^{\circ} \mathrm{C}$ for $72 \mathrm{~h}$. All isolates recovered were checked by morphological examination and aerotolerant experiments.

Genomic DNA was prepared from each isolate using the QIAamp DNA mini kit (Qiagen, Hilden, Germany) according to the manufacturer's instructions for Grampositive organisms. Isolates were confirmed as toxigenic C. difficile using PCR for tpi and $t c d B[7,8]$. Toxigenic $C$. difficile isolates were also screened for the enterotoxinencoding gene $t c d A$ and binary toxin genes $c d t A$ and $c d t B$ using multiplex PCR [7, 8]. Isolates recovered were considered ICU-acquired if the host patient had no toxigenic $C$. difficile in the swab collected within 2 days of admission.

\section{Strain typing}

Strain typing of toxigenic isolates was performed using multilocus sequence typing (MLST) as described previously [9]. Sequence types (STs) of C. difficile were also clustered into clades by phylogenetic analysis using the concatenated sequences of the seven loci of MLST [9]. For isolates belonging to the same ST, multiple-locus variable-number tandem repeat analysis (MLVA) was performed to further determine the relatedness of these isolates as described before [4]. The 6-loci MLVA scheme developed by Marsh J et al. [4, 10] was used and the exact size of amplicons was determined by capillary electrophoresis using an ABI 3730xl (Applied Biosystems, Carlsbad, CA).

\section{Statistical analysis}

Statistical analysis was performed using SPSS version 18.0 (SPSS Inc., Chicago, IL). Proportions were compared using a Chi-Squared test and a $P$ value $<0.05$ was considered statistically significant.

\section{Results}

During the 4-month period, a total of 396 adult patients were admitted to the ICU, among which rectal swabs were collected from 360 (90.9\%) patients within 2 days 
of admission to ICU (Fig. 1). Among the 360 patients, 314 had stayed in the ICU for at least 3 days, with rectal swabs collected from 231 (73.6 \%, 231/314) patients within the 3 days prior to ICU discharge (Fig. 1). Only 6 $(1.7 \%, 6 / 360)$ patients had toxigenic $C$. difficile on admission, while $10(4.3 \%, 10 / 231)$ had it at discharge. As none developed diarrhea during their hospitalization in ICU, all 16 patients positive for toxigenic C. difficile (either on admission or at discharge) were classified as carriers. Of the 6 carriers of toxigenic $C$. difficile on admission, 4 had a rectal swab collected within 3 days of discharge but toxigenic $C$. difficile was not detected. For all 10 carriers of toxigenic $C$. difficile at discharge, swabs collected on admission were negative, suggesting that they acquired toxigenic $C$. difficile during their ICU stay. The prevalence of colonization with toxigenic $C$. difficile on admission appeared to be higher in patients who were 65 or older $(3.3 \%, 4 / 122)$ than that in those who were younger $(0.8 \%, 2 / 238)$ but this difference was not statistically significant $(P=0.2)$. Most $(8 / 10)$ of patients acquired toxigenic $C$. difficile during their ICU stay were also 65 or older and had a higher rate of acquisition of toxigenic $C$. difficile $(9.9 \%, 8 / 81)$ than those who were younger $(1.3 \%, 2 / 150 ; P<0.05)$.

Toxigenic $C$. difficile isolates were recovered from all of the 16 carriers detected by PCR. Among these 16 toxigenic isolates, 10 were positive for $t c d A$ and $t c d B$ $(\mathrm{A}+\mathrm{B}+)$ and 6 were positive for $t c d B$ only $(\mathrm{A}-\mathrm{B}+)$. In addition, binary toxin genes were detected in one isolate, which was collected at discharge and was $\mathrm{A}+\mathrm{B}+$. The 6 isolates recovered from swabs collected on admission belonged to four STs, i.e. ST2 $(n=1)$, ST3 $(n=$ 2), ST6 $(n=1)$ and ST54 $(n=2)$ (Fig. 1 and Table 1$)$. In contrast, most (6 of 10) isolates recovered from swabs collected at discharge belonged to ST37, which was not detected on admission. The remaining four isolates recovered from swabs collected at discharge belonged to
ST2 $(n=1)$, ST54 $(n=2)$ and ST103 $(n=1)$ (Fig. 1 and Table 1). The isolate carrying binary toxin genes belonged to ST103.

During their ICU stay, 54 (15.0\%) out of 360 patients developed diarrhea, of which four cases $(1.1 \%, 4 / 360$; their ages ranged from 35 to 73 ) were CDI. The length of ICU stay was 3,744 ICU days for the 360 patients and CDI incidence was 10.7 cases per 10,000 ICU days. None of the four cases were positive for toxigenic $C$. difficile either on admission or at discharge. Toxigenic C. difficile was cultured from all four patients and belonged to ST3 $(n=1)$, ST37 $(n=2)$ or ST129 $(n=1)$ (Fig. 1 and Table 1).

In total, 20 toxigenic $C$. difficile isolates were recovered from either swabs or stool samples. The 20 isolates belonged to 7 STs (ST2, ST3, ST6, ST37, ST54, ST103 and ST129; Table 1). Toxigenic $C$. difficile could be clustered into four clades based on STs [9]. Among the 7 STs identified in this study, ST5 belongs to clade 3 and ST37 belongs to clade 4, while the remaining STs were of clade 1 (Table 1). As there were multiple isolates of ST2 $(n=2)$, ST3 $(n=2)$, ST37 $(n=8)$ and ST54 $(n=4)$, those belonging to the same ST were further typed using MLVA. However, none of these isolates had identical MLVA patterns (Fig. 1 and Table 1).

\section{Discussion}

Previous studies revealed that the prevalence of colonization with toxigenic $C$. difficile in adult hospitalized patients varied from $4.4 \%$ to $23.2 \%$ [6] with the pooled rate being $8.1 \%$ [5]. However, very few studies investigated the colonization of toxigenic $C$. difficile among ICU patients. In a study at Johns Hopkins Hospital, $3.1 \%$ of patients were colonized with toxigenic $C$. difficile on admission to ICU. This suggests that the colonization rate of toxigenic $C$. difficile in ICU patients may be lower than those described for the general

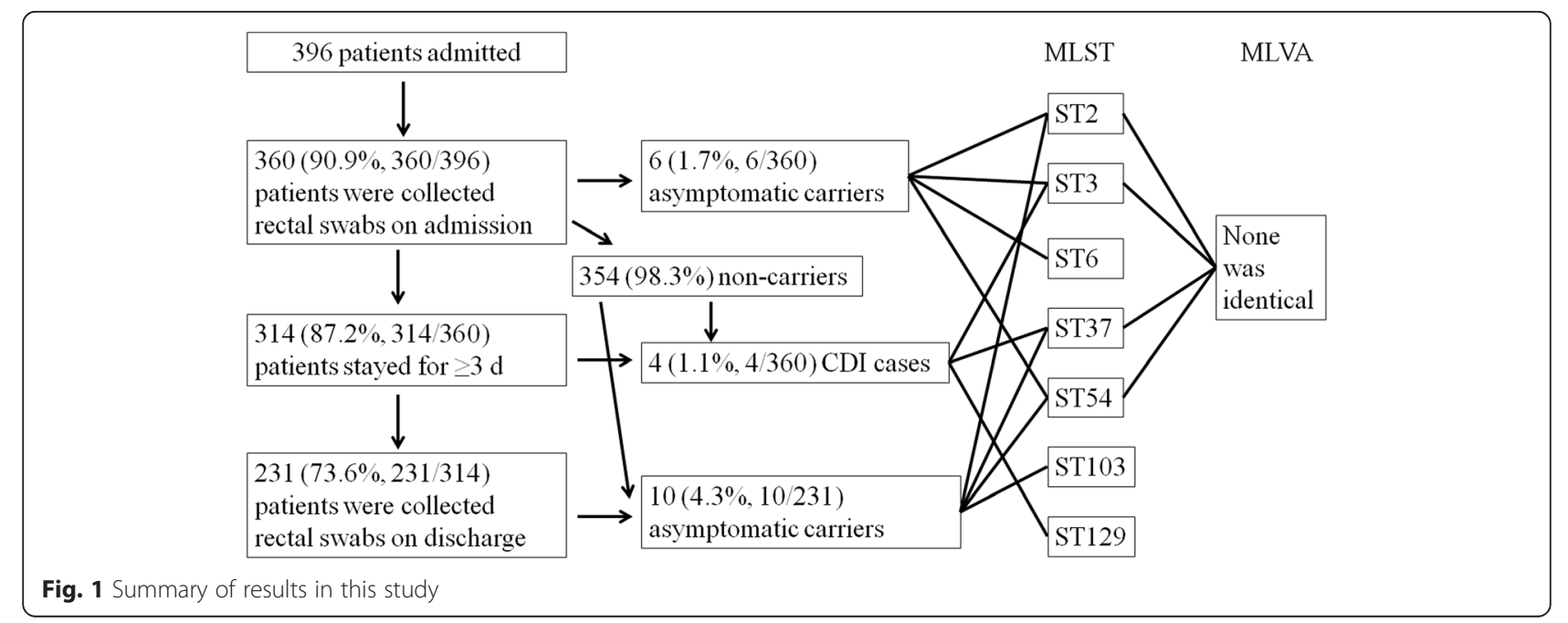


Table 1 Characteristics of patients with toxigenic C. difficile

\begin{tabular}{|c|c|c|c|c|c|c|}
\hline Patient & ICU stay, $\mathrm{d}$ & Diseases & Collection date & ST & Clade $^{a}$ & MLVA profile \\
\hline \multicolumn{7}{|c|}{ Isolates detected on admission } \\
\hline 1 & 13 & Cholangitis, bacteremia & November & 2 & 1/HA1 & $316 / 171 / 264 / 280 / 280 / 371$ \\
\hline 2 & 19 & Stroke & September & 3 & 1/HA1 & 259/187/329/365/365/117 \\
\hline 3 & 7 & $\begin{array}{l}\text { Systemic EB virus positive T lymphocyte } \\
\text { proliferative disease, pneumonia }\end{array}$ & September & 3 & $1 / \mathrm{HA} 1$ & $425 / 195 / 243 / 336 / 336 / 455$ \\
\hline 4 & 27 & Pneumonia, Guillain-Barré syndrome & November & 6 & 1/HA1 & ND \\
\hline 5 & 6 & Septic shock, colon cancer & August & 54 & 1/HA1 & $322 / 171 / 286 / 365 / 365 / 117$ \\
\hline 6 & 4 & Gastric cancer, pneumonia & September & 54 & $1 / \mathrm{HA} 1$ & $322 / 171 / 286 / 344 / 344 / 223$ \\
\hline \multicolumn{7}{|c|}{ Isolates detected on discharge } \\
\hline 7 & 12 & Pneumonia, uremia & October & 2 & 1/HA1 & 259/164/263/344/344/488 \\
\hline 8 & 12 & Embedded abdominal wall hernia & August & 37 & $4 / A-B+$ & $129 / 336 / 209 / 314 / 314 / 420$ \\
\hline 9 & 6 & Septic shock, ovian cancer & October & 37 & $4 / A-B+$ & 129/344/209/294/293/438 \\
\hline 10 & 10 & Brain glioma & October & 37 & $4 / A-B+$ & 129/336/209/307/307/421 \\
\hline 11 & 17 & Liver cirrhosis & November & 37 & $4 / A-B+$ & $129 / 367 / 209 / 300 / 300 / 438$ \\
\hline 12 & 32 & Pancreatitis & November & 37 & $4 / A-B+$ & 129/336/209/300/300/487 \\
\hline 13 & 5 & Gangrenous appendicitis, peritonitis & November & 37 & $4 / A-B+$ & 129/404/209/307/307/454 \\
\hline 14 & 10 & Pneumonia & August & 54 & $1 / \mathrm{HA} 1$ & 276/171/294/365/365/117 \\
\hline 15 & 29 & Anterior pituitary hypofunction & October & 54 & 1/HA1 & 218/187/294/414/414/117 \\
\hline 16 & 8 & Pneumonia & September & 103 & 1/HA1 & ND \\
\hline \multicolumn{7}{|c|}{ Isolates causing CDI } \\
\hline 17 & 20 & Pancreatitis & September & 3 & $1 / \mathrm{HA} 1$ & 292/148/337/351/351/117 \\
\hline 18 & 12 & Colon cancer & September & 37 & $4 / A-B+$ & $129 / 367 / 209 / 307 / 307 / 454$ \\
\hline 19 & 19 & Liver cirrhosis & November & 37 & $4 / A-B+$ & $129 / 367 / 209 / 300 / 300 / 420$ \\
\hline 20 & 29 & Liver cirrhosis & September & 129 & $1 / \mathrm{HA} 1$ & ND \\
\hline
\end{tabular}

${ }^{a}$ Names of clades are from reference [9]. ${ }^{\mathrm{b}} \mathrm{MLVA}$ profile: amplicon size (bp) for loci CDA6, CDG8, CDR5, CDR48, CDR49 and CDR60

population due to as yet unknown reasons [11]. We cannot rule out that the low prevalence of colonization with toxigenic $C$. difficile on admission to ICU may be due to the use of a rectal swab rather than stool. However, prior studies have demonstrated that rectal swabs and stool cultures were equivalent for detection of $C$. difficile in stool of CDI patients [12, 13]. Nonetheless, the low prevalence $(1.7 \%)$ of colonization on admission to ICU in our study was even lower than the $3.1 \%$ in study from Johns Hopkins Hospital, which also used rectal swabs for screening. The differences in colonization rate among studies remain unclear but may reflect the population risk of colonization with toxigenic C. difficile as previous studies have demonstrated that the prevalence of colonization with toxigenic $C$. difficile varies significantly by geographic location [6]. Although the prevalence of toxigenic $C$. difficile in China remains still largely unknown, it is possible that the prevalence of colonization with toxigenic $C$. difficile in China is truly lower than that in western countries. This may not be a surprise as there are significant differences in food and drinking habits between China and western countries.
However, large-scale multi-center studies are required to fully characterize the prevalence of toxigenic $C$. difficile in China.

A systematic review and meta analysis found that patients colonized with toxigenic $C$. difficile on hospital admission were 6 times more likely to develop CDIs compared with noncolonized patients [5]. In the previous study on ICU patients, colonization with toxigenic C. difficile on admission and colonization during ICU stay have also been identified as an independent risk factors for subsequent CDI with relative risks being 8.62 and 10.93, respectively [11]. However, it has also been thought that colonization with $C$. difficile could be protected from subsequent CDI [14]. In this study, none of the six patients colonized with toxigenic $C$. difficile on admission and none of the ten patients colonized at discharge developed diarrhea during their ICU stay. Patients who were 65 years of age or older had a much higher risk of developing CDI than younger patients [15]. Our study found that patients who were 65 or older were also more likely to acquire toxigenic $C$. difficile and become asymptomatic carriers during their ICU stay. 
Active screening of elderly patients was therefore able to identify more carriers of toxigenic $C$. difficile and was more resource-efficient than screening all patients. However, the significance of active screening in clinical management and infection control needs to be justified as none of these elderly carriers developed CDI in our setting. Our findings do not support the active screening of all patients for toxigenic $C$. difficile on admission to ICU, in the setting of a low prevalence of colonization, to identify patients in high risks of subsequent CDI. More studies are warranted to confirm whether colonization with toxigenic $C$. difficile is truly a high-risk factor for subsequent CDI in ICU patients.

None of the four patients colonized with a toxigenic $C$. difficile had CDI at discharge. A previous study reported that $\beta$-lactam- $\beta$-lactamase inhibitor combinations and metronidazole were associated with a loss of $C$. difficile colonization [16]. During their ICU stay, three of the four patients received either piperacillin-tazobactam $(n=$ 2 ) or cefoperazone-sulbactam $(n=1)$ and the remaining patient received metronidazole.

Ten patients had toxigenic C. difficile at discharge and four patients developed CDI during their ICU stay. The fourteen patients did not have toxigenic $C$. difficile on admission, suggesting that their toxigenic $C$. difficile were acquired during their ICU stay. An obvious concern is whether these ICU-acquired isolates were from those introduced to ICU on admission. However, the majority (10/14) of ICU-acquired toxigenic C. difficile belonged to STs, which were not detected on admission. The remaining four ICU-acquired isolates ( 2 of ST54, 1 of ST2 and 1 of ST3) matched those detected on admission with the same ST. However, MLVA analysis revealed that none of these paired ICU-acquired and introduced isolates were identical. Therefore, there was no evidence that the ICU-acquisitions were from those introduced isolates. This unexpected finding suggests that there were additional sources (e.g. healthcare workers, visiting family members or the environment) of toxigenic $C$. difficile in ICU, which is yet to be determined. Our findings do not support the active screening of all patients for toxigenic $C$. difficile on admission to ICU in our setting to identify the potential source of subsequent CDI. In a setting with low prevalence of colonization, active screening for toxigenic C. difficile may not be justified as a resource-efficient measure for the prevention of CDI.

Most of the ICU-acquired toxigenic $C$. difficile isolates (8/14) belonged to a single ST, ST37, suggesting possible transmission of a certain clone in ICU. However, none of the 8 ST37 isolates were identical by MLVA, which does not support the hypothesis of the transmission of a certain clone. Alternatively, although MLVA has been widely adopted for strain typing and has been successfully used for C. difficile [4], it may be too discriminatory to define clones, which has been observed by other investigators [17-19]. It is therefore possible that MLVA may not be an ideal method for investigating the transmission of toxigenic $C$. difficile. Molecular typing is critical to track the transmission of toxigenic C. difficile within hospitals but none of the currently-used typing methods, including pulsed field gel electrophoresis (PFGE), restriction enzyme analysis (REA), MLST and PCR ribotyping, is ideal for $C$. difficile [20]. Although a previous study found that MLVA and whole genome sequencing had similar discriminatory ability [21], whole genome sequencing may still be required to untangle whether there was clonal transmission of toxigenic $C$. difficile in ICU.

We found a binary toxin-producing $C$. difficile isolate, which colonized (rather than caused CDI) a patient on admission to ICU. Recent publications showed that binary toxin is a marker for highly virulent $C$. difficile or contributes directly to the virulence [22]. The identification of a colonizing binary toxin-positive isolate in this study highlights that the presence of binary toxin genes does not necessarily indicate diarrhea, which also depends on host factors.

CDI incidence (10.7 cases per 10,000 ICU days) in this study was lower than that (25.2 cases per 10,000 ICU days) of our previous study, which was performed between May 2012 and January 2013 in the same unit [23]. This could be due to improved infection control practices in the unit. For instance, hand hygiene compliance of healthcare workers in the unit had increased from $49.5 \%$ in 2012 to $79.7 \%$ in 2014. Ongoing monitoring of CDI incidence is required to examine whether the reduction of CDI continues.

Our study has several limitations. First, this was a single center study and we were not able to collect swabs from all eligible patients and some additional carriers might have been missed. Second, this study was carried out to explore the prevalence of colonization with and the transmission of toxigenic C. difficile in ICU, we did not specifically investigate the risk factors for the acquisition of toxigenic $C$. difficile in ICU. In addition, the small number of patients colonized with toxigenic C. difficile may result in our study being underpowered for the detection of risk factors for colonization. Third, we did not follow up the carriers who were positive at discharge from ICU and therefore we were unable to find out whether these colonizers developed diarrhea later on. Without follow up we were also unable to determine whether the colonization of toxigenic $C$. difficile was transient or could persist for a period. Fourth, as previous studies have demonstrated that PCR assays may be less sensitive than toxigenic culture for detection of asymptomatic carriage of toxigenic C. difficile $[12,13]$, some carriers might have been missed in this study. 


\section{Conclusions}

The prevalence of colonization with toxigenic $C$. difficile and the incidence of CDI among patients on admission to ICU were low in our settings. Although some patients (4.3\%) acquired toxigenic $C$. difficile and became asymptomatic carriers during their ICU stay, these ICUacquired isolates were not genetically linked with those carried by patients at admission. Active screening for toxigenic $C$. difficile may not be a resource-efficient measure for the prevention of CDI in such a setting with a low prevalence of colonization. Importantly, these conclusions may be not generalizable due to the low prevalence of $C$. difficile colonization and CDI in our patient population.

\section{Abbreviations}

CDI, C. difficile infection; MLST, multi-locus sequence typing; MLVA, multiplelocus variable number tandem repeat analysis; ST, sequence type

\section{Acknowledgments}

We are grateful for Dr. Andrew Ginn at Centre for Infectious Diseases and Microbiology, The Westmead Institute for Medical Research, The University of Sydney, Sydney, NSW, Australia for his critical review for this paper.

\section{Funding}

This study was supported by the New Century Excellent Talents Program, Ministry of Education, China (project no. NCET-13-0399) and grants from Sichuan provincial Science and Technology Agency, China (project no. $2011 F Z 0111$ and 2015SZ0234-4). Ministry of Education, China and Sichuan provincial Science and Technology Agency.

\section{Availability of data and materials}

Data supporting the findings are included in the manuscript.

\section{Authors' contributions}

$X Z, X W, J Y, X L, C L$ carried out the study and helped to draft the manuscript. $X W$ and $Z Z$ conceived of the study, analyzed the data and drafted the manuscript. All authors read and approved the final manuscript.

\section{Competing interests}

The authors declare that they have no competing interests.

\section{Consent for publication}

Not applicable.

\section{Ethics approval and consent to participate}

This study was approved by the Ethics Committee of West China Hospital. The need of patient consents was waived by the Ethics Committee.

\section{Author details \\ ${ }^{1}$ Center of Infectious Diseases, West China Hospital, Sichuan University, Guoxuexiang 37, Chengdu 610041, China. ²Division of Infectious Diseases, State Key Laboratory of Biotherapy, Chengdu, China. ${ }^{3}$ Department of Infection Control, West China Hospital, Sichuan University, Chengdu, China. ${ }^{4}$ Intensive Care Unit, West China Hospital, Sichuan University, Chengdu, China. ${ }^{5}$ Department of Clinical Microbiology, Xindu District Hospital, Chengdu, China.}

Received: 1 December 2015 Accepted: 20 July 2016

Published online: 09 August 2016

\section{References}

1. Leffler DA, Lamont JT. Clostridium difficile Infection. N Engl J Med. 2015;373:287-8.

2. Kundrapu S, Sunkesula VC, Jury LA, Sethi AK, Donskey CJ. Utility of perirectal swab specimens for diagnosis of Clostridium difficile infection. Clin Infect Dis. 2012;55:1527-30.
3. Vonberg RP, Kuijper EJ, Wilcox MH, Barbut F, Tull P, Gastmeier P, European Cd-ICG, European Centre for Disease P, Control, van den Broek PJ, et al. Infection control measures to limit the spread of Clostridium difficile. Clin Microbiol Infect. 2008;14 Suppl 5:2-20.

4. Curry SR, Muto CA, Schlackman JL, Pasculle AW, Shutt KA, Marsh JW, Harrison LH. Use of multilocus variable number of tandem repeats analysis genotyping to determine the role of asymptomatic carriers in Clostridium difficile transmission. Clin Infect Dis. 2013;57:1094-102.

5. Zacharioudakis IM, Zervou FN, Pliakos EE, Ziakas PD, Mylonakis E. Colonization with toxinogenic C. difficile upon hospital admission, and risk of infection: a systematic review and meta-analysis. Am J Gastroenterol. 2015;110:381-90. quiz 391.

6. Hung YP, Lee JC, Lin HJ, Liu HC, Wu YH, Tsai PJ, Ko WC. Clinical impact of Clostridium difficile colonization. J Microbiol Immunol Infect. 2015;48:241-8

7. Lemee L, Dhalluin A, Testelin S, Mattrat MA, Maillard K, Lemeland JF, Pons $J$. Multiplex PCR targeting tpi (triose phosphate isomerase), tcdA (Toxin A), and $t c d B$ (Toxin B) genes for toxigenic culture of Clostridium difficile. J Clin Microbiol. 2004:42:5710-4.

8. Persson S, Torpdahl M, Olsen KE. New multiplex PCR method for the detection of Clostridium difficile toxin $A(t c d A)$ and toxin $B(t c d B)$ and the binary toxin $(c d t A / c d t B)$ genes applied to a Danish strain collection. Clin Microbiol Infect. 2008;14:1057-64.

9. Griffiths D, Fawley W, Kachrimanidou M, Bowden R, Crook DW, Fung R, Golubchik T, Harding RM, Jeffery KJ, Jolley KA, et al. Multilocus sequence typing of Clostridium difficile. J Clin Microbiol. 2010;48:770-8.

10. Marsh JW, O'Leary MM, Shutt KA, Sambol SP, Johnson S, Gerding DN, Harrison LH. Multilocus variable-number tandem-repeat analysis and multilocus sequence typing reveal genetic relationships among Clostridium difficile isolates genotyped by restriction endonuclease analysis. J Clin Microbiol. 2010:48:412-8

11. Tschudin-Sutter S, Carroll KC, Tamma PD, Sudekum ML, Frei R, Widmer AF, Ellis BC, Bartlett J, Perl TM. Impact of toxigenic Clostridium difficile colonization on the risk of subsequent $C$. difficile infection in intensive care unit patients. Infect Control Hosp Epidemiol. 2015;36:1324-9.

12. Marsh JW, Arora R, Schlackman JL, Shutt KA, Curry SR, Harrison LH. Association of relapse of Clostridium difficile disease with BI/NAP1/027. J Clin Microbiol. 2012:50:4078-82

13. Donskey CJ, Sunkesula VC, Jencson AL, Stone ND, Gould CV, McDonald LC, Samore M, Mayer J, Pacheco S, Sambol S, et al. Utility of a commercial PCR assay and a clinical prediction rule for detection of toxigenic Clostridium difficile in asymptomatic carriers. J Clin Microbiol. 2014;52:315-8.

14. Galdys AL, Curry SR, Harrison LH. Asymptomatic Clostridium difficile colonization as a reservoir for Clostridium difficile infection. Expert Rev Anti Infect Ther. 2014;12:967-80.

15. Pepin J, Valiquette L, Cossette B. Mortality attributable to nosocomial Clostridium difficile-associated disease during an epidemic caused by a hypervirulent strain in Quebec. CMAJ. 2005;173:1037-42.

16. Dubberke ER, Reske KA, Seiler S, Hink T, Kwon JH, Burnham CA. Risk Factors for Acquisition and Loss of Clostridium difficile Colonization in Hospitalized Patients. Antimicrob Agents Chemother. 2015;59:4533-43.

17. Tanner HE, Hardy KJ, Hawkey PM. Coexistence of multiple multilocus variable-number tandem-repeat analysis subtypes of Clostridium difficile PCR ribotype 027 strains within fecal specimens. J Clin Microbiol. 2010:48:985-7.

18. Killgore G, Thompson A, Johnson S, Brazier J, Kuijper E, Pepin J, Frost EH, Savelkoul P, Nicholson B, van den Berg RJ, et al. Comparison of seven techniques for typing international epidemic strains of Clostridium difficile: restriction endonuclease analysis, pulsed-field gel electrophoresis, PCR-ribotyping, multilocus sequence typing, multilocus variable-number tandem-repeat analysis, amplified fragment length polymorphism, and surface layer protein A gene sequence typing. J Clin Microbiol. 2008;46:431-7.

19. Dawson LF, Valiente E, Wren BW. Clostridium difficile-a continually evolving and problematic pathogen. Infect Genet Evol. 2009;9:1410-7.

20. Knetsch CW, Lawley TD, Hensgens MP, Corver J, Wilcox MW, Kuijper EJ. Current application and future perspectives of molecular typing methods to study Clostridium difficile infections. Euro Surveill. 2013;18:20381.

21. Eyre DW, Fawley WN, Best EL, Griffiths D, Stoesser NE, Crook DW, Peto TE, Walker AS, Wilcox MH. Comparison of multilocus variable-number tandem- 
repeat analysis and whole-genome sequencing for investigation of Clostridium difficile transmission. J Clin Microbiol. 2013;51:4141-9.

22. Bacci S, Molbak K, Kjeldsen MK, Olsen KE. Binary toxin and death after Clostridium difficile infection. Emerg Infect Dis. 2011;17:976-82.

23. Wang X, Cai L, Yu R, Huang W, Zong Z. ICU-Onset Clostridium difficile infection in a university hospital in China: a prospective cohort study. PLoS One. 2014;9:e111735.

Submit your next manuscript to BioMed Central and we will help you at every step:

- We accept pre-submission inquiries

- Our selector tool helps you to find the most relevant journal

- We provide round the clock customer support

- Convenient online submission

- Thorough peer review

- Inclusion in PubMed and all major indexing services

- Maximum visibility for your research

Submit your manuscript at www.biomedcentral.com/submit 